\title{
Negative feedback effects on star formation history and cosmic reionization
}

\author{
L. Wang ${ }^{1}$, J. Mao ${ }^{2}$, S. Xiang ${ }^{1}$, and Y.-F. Yuan ${ }^{1}$ \\ 1 Center for Astrophysics, University of Science and Technology of China, Hefei, 230026, PR China \\ e-mail: ws12008@mail.ustc.edu.cn \\ 2 Yunnan Observatory, National Astronomical Observatories, Chinese Academy of Sciences, PO Box 110, Kunming, \\ Yunnan Province, 650011, PR China \\ e-mail: jirongmao@ynao.ac.cn; [spxiang;yfyuan]@ustc.edu.cn
}

Received 6 April 2008 / Accepted 18 November 2008

\begin{abstract}
Context. The mechanical and radiative feedback that exists in the star formation history affects the subsequent star formation rate. Aims. After considering the effects of negative feedback on the process of star formation, we explore the relationship between star formation process and the associated feedback, by investigating how the mechanical feedback from supernovae( $\mathrm{SNe})$ and radiative feedback from luminous objects regulate the star formation rate and therefore affect the cosmic reionization.

Methods. Based on our present knowledge of the negative feedback theory and some numerical simulations, we construct an analytic model in the framework of the Lambda cold dark matter model. In certain parameter regions, our model can explain some observational results properly.

Results. In large halos $\left(T_{\mathrm{vir}}>10^{4} \mathrm{~K}\right)$, both mechanical and radiative feedback have a similar behavior: the relative strength of negative feedback reduces as the redshift decreases. In contrast, in small halos $\left(T_{\text {vir }}<10^{4} \mathrm{~K}\right)$ that are thought to breed the first stars at early time, the radiative feedback gets stronger when the redshift decreases. And the star formation rate in these small halos depends very weakly on the star-formation efficiency.

Conclusions. Our results show that the radiative feedback is important for the early generation stars. It can suppress the star formation rate considerably. But the mechanical feedback from the SNe explosions is not able to affect the early star formation significantly. The early star formation in small-halo objects is likely to be self-regulated. The radiative and mechanical feedback dominates the star formation rate of the PopII/I stars all along. The feedback from first generation stars is very strong and should not be neglected. However, their effects on the cosmic reionization are not significant, which results in a small contribution to the optical depth of Thomson scattering.
\end{abstract}

Key words. methods: analytical - cosmology: theory - Galaxy: evolution - stars: formation

\section{Introduction}

In the past decades, the so-called "bottom-up" hierarchical scenario for the large-scale structure formation in the Lambda cold dark matter $(\Lambda \mathrm{CDM})$ cosmogonies has been getting decisive support from more and more observations (e.g. from HST, WMAP, SDSS) and from high-resolution $N$-body/hydrodynamics numerical simulations. Owing to the complexity of the baryonic evolution in the radiative background and gravitational field, the galaxy formation and evolution in dark matter halos and the reionization history of IGM have not been fully understood yet. The difficulties stem from our lack of knowledge of those early formed objects that are unobservable at present. The formation of the first objects is relevant to the cosmic reionization history. Recent detections of the Gunn-Peterson trough (Gunn \& Peterson 1965) in the spectra of QSOs with $z \simeq 6$ indicate less $50 \%$ neutral hydrogen at $z \sim 6.5$ (Fan et al. 2006; Wyithe et al. 2005). The ongoing observations by the WMAP satellite of cosmic microwave background $(\mathrm{CMB})$ and the highest redshift QSOs put very tight constraints on the reionization history of the Universe. The WMAP five-year observation manifests the Thomson scattering optical depth, $\tau_{\mathrm{e}}=0.084_{-0.016}^{+0.016}$ (Komatsu et al. 2008), which suggests that our Universe might be reionized during the period of redshift $9.4 \leq z_{\text {re }} \leq 12.2$.

It is well-known that chemical elements heavier than lithium are produced exclusively through stellar nucleosynthesis. Some of the first generation stars (the so-called population III stars, hereafter, PopIII) die as SNe explosions, which can expel heavier elements into the intergalactic medium (IGM). When the metal elements in IGM are enriched to a certain threshold $Z_{\text {crit }}$, the population II/I stars (hereafter PopII/I) will form and take the place of the first stars to light the universe. Thence, the $\mathrm{SNe}$ from PopIII stars determine the transition from PopIII to PopII/I. The existence of PopIII stars can help in explaining the metal enrichment from $Z \simeq 10^{-12}-10^{-10}$ to the lowest metallicity of PopII stars $Z \simeq 10^{-4}-10^{-3}$, the formation of massive black holes, the reionization of the universe, the starting engine for the formation of the first galaxies and the G-dwarf, and so on (Ciardi \& Ferrara 2005). But there is still no hope in observing the first generation stars until the launch of the James Webb Space Telescope (JWST), the successor of the Hubble Space 
Telescope (HST) (Barkana \& Loeb 2001). JWST is expected to find the pair instability SNe (PISNe) from massive PopIII stars (Wise \& Abel 2005).

Due to the absence of the observational data of very highredshift $(z \geq 10)$ objects, theoretical investigations are mainly based on numerical simulations. Some works concentrated on the effects of the first-generation $\mathrm{SNe}$ explosions (Yoshida et al. 2003; Kitayama \& Yoshida 2005; Greif et al. 2007), while the others focused on the strong stellar and galactic winds from PopIII stars (Meynet et al. 2006; Ricotti et al. 2008). Based on these works, we put forward a model to describe the global effects from the PopIII stars at an early time, such as the SFR density, the IGM reionization, and so on.

In this paper, we study the effects of the mechanical feedback from $\mathrm{SNe}$ and the radiative feedback from stars and UV background, especially the negative feedback effects on the SFH and the cosmic reionization history. The radiative feedback from PopIII stars includes the ISM photoevaporation and IGM reionization. The outline of this paper is as follows. In Sect. 2, we describe the evolution of dark matter halos in the $\Lambda$ CDM model. Within this framework, the SFR in a halo can be expressed as an analytic formula with some free parameters. Mechanical feedback from stars is studied in Sect. 3. In Sect. 4, we introduce an analytic model to deal with the radiative feedback from PopIII. Finally, our discussion and conclusions are presented in the last section. Throughout this paper, we adopt the cosmological parameters consistent with the 5 years WMAP data: $\Omega_{\mathrm{m}} h^{2}=0.1358, \Omega_{\Lambda}=0.726, \Omega_{\mathrm{b}}=0.0456, h=0.7, \sigma_{8}=0.812$ (Komatsu et al. 2008).

\section{Cold dark matter model and star formation in galaxies}

\subsection{Redshift evolution of dark matter halos}

In the hierarchical clustering scenario of CDM halos, a simple and successful model for the formation and distribution of spheroidal or ellipsoidal collapsing objects was presented in last century based on the theory of Gaussian random fields about cosmological density perturbation (Press \& Schechter 1974; Bardeen et al. 1986). In this scenario, gravity governs almost the whole process. The dark matter halos increase their own mass through accreting matter and merging with each other (Lacey \& Cole 1993). Based on this model, Sasaki (1994) proposed an analytic formula to describe the formation and evolution of the CDM halos. In this formalism, the number density of collapsed objects with mass in the range $(M, M+\mathrm{d} M)$, which are formed at the redshift interval $\left(z_{\mathrm{c}}, z_{\mathrm{c}}+\mathrm{d} z_{\mathrm{c}}\right)$ and survive till redshift $z$, is (Sasaki 1994; Chiu \& Ostriker 2000),

$$
\begin{aligned}
N\left(M, z, z_{\mathrm{c}}\right) \mathrm{d} M \mathrm{~d} z_{\mathrm{c}}= & \alpha N_{M}\left(z_{\mathrm{c}}\right)\left(\frac{\delta_{\mathrm{c}}}{D\left(z_{\mathrm{c}}\right) \sigma(M)}\right)^{2} \frac{\dot{D}\left(z_{\mathrm{c}}\right)}{D(z)} \\
& \times \frac{\mathrm{d} z_{\mathrm{c}}}{H\left(z_{\mathrm{c}}\right)\left(1+z_{\mathrm{c}}\right)} \mathrm{d} M .
\end{aligned}
$$

Here the overdot represents the time derivative, and $N_{M}\left(z_{\mathrm{c}}\right) \mathrm{d} M$ is the number of collapsed objects per unit comoving volume within a mass range $(M, M+\mathrm{d} M)$ at redshift $z_{\mathrm{c}}$ (Press \& Schechter 1974). In Eq. (1), $\delta_{\mathrm{c}}$ is a constant, usually taken to be 1.686 in a matter-dominated flat universe $\left(\Omega_{\mathrm{m}}=1\right)$. This value is quite insensitive to the cosmological parameters (Eke et al. 1996), where $H(z)$ is the Hubble parameter, $D(z)$ the growth factor for linear perturbations, and $\sigma(M)$ the rms mass fluctuation on a mass scale $M$. In addition, $N\left(M, z, z_{\mathrm{c}}\right)$ in Eq. (1) represents the formation rate of halos weighted by their survival probability. For more accuracy, here we use Sheth \& Tormen (1999)'s modified formula for the expression of $N_{M}\left(z_{\mathrm{c}}\right) \mathrm{d} M$, which fits the numerical simulations better than the original one, especially at high redshift. Numerical simulations also indicate $\alpha=0.707$ (see Sheth et al. 2001, for more details).

\subsection{Star-formation rate in galaxies and the cosmic SFR density}

Stars or even galaxies are confirmed to form through cooling and condensation of baryons within the DM halos (White $\&$ Rees 1978). The formation and evolution of galaxies and the associated star formation histories have been studied extensively via both numerical simulations and semi-analytic models (Cen \& Ostriker 1992; Chiu \& Ostriker 2000; Choudhury \& Srianand 2002; Springel \& Hernquist 2003; Nagamine et al. 2006). Following Cen \& Ostriker (1992), Chiu \& Ostriker (2000) and Choudhury \& Srianand (2002), we assume ${ }^{1}$ that the SFR in a halo with mass $M$ at $z$ that has collapsed at an earlier redshift $z_{\mathrm{c}}$, is given by

$\dot{M}_{\mathrm{SF}}\left(M, z, z_{\mathrm{c}}\right)=f_{*} M \frac{\Omega_{\mathrm{b}}}{\Omega_{\mathrm{m}}} \times \frac{t(z)-t\left(z_{\mathrm{c}}\right)}{\kappa^{2} t_{\mathrm{dyn}}^{2}\left(z_{\mathrm{c}}\right)} \exp \left(\frac{t(z)-t\left(z_{\mathrm{c}}\right)}{-\kappa t_{\mathrm{dyn}}\left(z_{\mathrm{c}}\right)}\right)$.

Here, $f_{*}, t(z), t_{\text {dyn }}$ are the fraction of total baryonic mass in a halo that will be converted to stars, the age of the Universe at redshift $z$, and the dynamical time scale, respectively. In our model, we take $f_{*}^{\text {III }}=0.07$ (Choudhury \& Ferrara 2006) for PopIII stars and $f_{*}^{\mathrm{II}}=0.3$ for PopII/I stars. The dynamical time scale $t_{\mathrm{dyn}}$ is given by (Chiu \& Ostriker 2000),

$t_{\text {dyn }}(z)=\sqrt{\frac{3 \pi}{32 G \rho_{\text {vir }}(z)}}$,

and here,

$\rho_{\mathrm{vir}}(z)=\Delta_{\mathrm{c}}(z) \rho_{\mathrm{c}}(z)$,

$\Delta_{\mathrm{c}}(z)=18 \pi^{2}+82 d(z)-39 d^{2}(z)$,

$d(z)=\frac{\Omega_{\mathrm{m}}(1+z)^{3}}{\Omega_{\mathrm{m}}(1+z)^{3}+\Omega_{\Lambda}}-1$,

$\rho_{\mathrm{c}}(z)=\frac{3 H^{2}(z)}{8 \pi G}$.

The duration of star formation activity in a halo depends on the value of $\kappa$. Note that $\kappa \rightarrow 0$ corresponds to the star formation occurring in a single burst. By using a 3D cosmological code that includes most of the needed physics to simulate the formation and evolution of the first galaxies, Ricotti et al. (2002a,b, 2008) show that the first luminous objects ("small-halo objects") are characterized by "bursting" star formation. In this paper, we thus set $\kappa_{\mathrm{III}}=0.01$ for PopIII SF (Samui et al. 2007) and $\kappa_{\mathrm{II}}=1$ for PopII/I (Cen \& Ostriker 1992; Chiu \& Ostriker 2000; Choudhury \& Srianand 2002).

Consequently, the cosmic SFR per unit comoving volume (SFR density, hereafter SFRD) at a redshift $z$ can be written as

$\dot{\rho}_{\mathrm{SF}}(z)=\int_{z}^{\infty} \mathrm{d} z_{\mathrm{c}} \int_{M_{\text {low }}}^{\infty} N\left(M^{\prime}, z, z_{\mathrm{c}}\right) \times \dot{M}_{\mathrm{SF}}\left(M^{\prime}, z, z_{\mathrm{c}}\right) \mathrm{d} M^{\prime}$,

\footnotetext{
1 In fact, this is not the most fundamental assumption (see Appendix A for details).
} 
where the lower mass cutoff $M_{\text {low }}(z)$ at a given epoch depends on the cooling efficiency of the gas and the different feedback processes. Following Barkana \& Loeb (2005), the lower mass cutoff can be expressed as

$M_{\text {low }}(z)=9.2 \times 10^{7} M_{\odot}\left(\frac{V_{\mathrm{c}}}{16.5 \mathrm{~km} \mathrm{~s}^{-1}}\right)^{3}\left(\frac{1+z}{10}\right)^{-\frac{3}{2}}$,

where the circular velocity $V_{\mathrm{c}}=\left(2 k_{\mathrm{B}} T_{\mathrm{vir}} / \mu m_{\mathrm{p}}\right)^{1 / 2}$, the mean molecular weight $\mu=0.61$, and $T_{\text {vir }}$ is the virial temperature of halos. Halos with $T_{\text {vir }} \geq 10^{4} \mathrm{~K}$ are able to cool via atomic transitions. It is usually considered as standard in most semianalytic models that the minimum mass of star-forming halos is $M_{\text {low }}(z=6) \sim 10^{8} M_{\odot}$. When the $\mathrm{H}_{2}$ content of the gas is considerable, molecular line cooling can make gas condense within the small halos $\left(T_{\text {vir }} \sim 10^{3} \mathrm{~K}\right)$ and eventually form stars (Tegmark et al. 1997; Haiman et al. 2000; Barkana \& Loeb 2005; Schneider et al. 2006; Samui et al. 2007). In the $\Lambda$ CDM model, the number of halos with masses $10^{5}-10^{8} M_{\odot}\left(T_{\text {vir }}<10^{4} \mathrm{~K}\right)$ that are expected to possess PopIII stars at high-redshift decreases after $z \sim 10$ due to the DM halos merging process (Mo \& White 2002), so these small-halo objects can dominate the galaxy mass function until at least redshift $z \sim 10$ (Ricotti et al. 2002b). Finally, we consider the atomic cooling model $\left(T_{\mathrm{vir}} \sim 10^{4} \mathrm{~K}\right)$ for PopII/I and molecular cooling model $\left(T_{\text {vir }} \sim 10^{3} \mathrm{~K}\right)$ for PopIII. It is notable that the $M_{\text {low }}$ mentioned above corresponds to the neutral regions only.

On the other hand, the Lyman-Werner (912-1108 $\AA$ ) background can also inhibit the star formation (Haiman et al. 1997; Ricotti et al. 2002a; Yoshida et al. 2003). However, it is interesting that the positive feedback of $\mathrm{H}_{2}$ re-formation (e.g. in front of HII regions and inside relic HII regions) can counterbalance the negative feedback from LW background (Ricotti et al. 2001, 2002a). As a consequence, the formation of small-mass galaxies is not suppressed, so in this paper we do not consider the LW background feedback by assuming that positive feedback dominates and star formation in small galaxies is not suppressed.

\subsection{Poplll mass fraction}

With the evolution of the Universe, the characteristics of star formation changes from a metal-free, massive-star-dominated (PopIII) mode to a metal-enriched, normal-star-dominated (PopII/I) mode. Numerical simulations indicate that this transition occurs when the metal has been enriched to a critical value, $Z_{\text {crit }} \simeq 10^{-3.5 \sim-4} Z_{\odot}$ (Bromm et al. 2003; Smith \& Sigurdsson 2007). If most of the first generation stars die as PISNe, the volume-averaged mean metallicity would reach $Z \sim 10^{-4} Z_{\odot}$ at a redshift of $\sim 12-16$ (Yoshida et al. 2004). It is suggested that the PopIII stars are terminated at $z_{\text {end }} \sim 9-10$ (Ricotti et al. 2002b; Salvaterra \& Ferrara 2003; Matsumoto et al. 2005; Choudhury \& Ferrara 2006).

Based on the above discussions, we assume a PopIII mass fraction, $F_{\mathrm{III}}(z)$, which means the mass fraction of objects forming from gas with $Z<Z_{\text {crit }}$ at a redshift $z$, i.e. the sites of PopIII star formation (Scannapieco et al. 2003). Here $F_{\text {III }}(z)$ is merely a function of redshift $z$. The transition from PopIII to PopII/I should not happen suddenly at a certain redshift, because chemical feedback is a local process: with regions close to star formation sites rapidly becoming metalpolluted and overshooting $Z_{\text {crit }}$, and others remaining essentially metal-free. PopIII and PopII star formation modes could have been coeval (Ciardi \& Ferrara 2005), so instead of assigning $F_{\text {III }}(z)$ a Heaviside function $\theta\left(z-z_{\text {tran }}\right)$, here we introduce a
Table 1. Model parameters.

\begin{tabular}{rl}
\hline \hline Parameters & Referrence values \\
\hline$\epsilon_{\mathrm{SN}}^{\mathrm{III}}$ & 0.05 \\
$\epsilon_{\mathrm{SN}}^{\mathrm{II}}$ & 0.02 \\
$f_{\text {esc }}^{\mathrm{II}}$ & 0.8 \\
$f_{\text {esc }}^{\mathrm{II}}$ & 0.1 \\
$f_{*}^{\mathrm{III}}$ & 0.07 \\
$f_{*}^{\mathrm{II}}$ & 0.3 \\
\hline
\end{tabular}

function: $F_{\mathrm{III}}(\beta, z)=\left(z / z_{\operatorname{tran}}\right)^{\beta} /\left[1+\left(z / z_{\operatorname{tran}}\right)^{\beta}\right]$, in which $\beta$ is a free parameter describing the transition speed. The $\beta \rightarrow \infty$ limit reproduces the sharp transition case. In this work, we take the transition redshift to be $z_{\text {tran }} \simeq 14$, and the free parameter $\beta=12$, which ensures the mass fraction of PopIII is lower than 5 percent at $z_{\text {end }}$. Now the SFRD can be rewritten as

$$
\begin{aligned}
\dot{\rho}_{\mathrm{III}}(z)= & \int_{z}^{\infty} \mathrm{d} z_{\mathrm{c}} \int_{M_{\text {low }}}^{\infty} \mathrm{d} M^{\prime} N\left(M^{\prime}, z, z_{\mathrm{c}}\right) \\
& \times \dot{M}_{\mathrm{SF}}\left(M^{\prime}, z, z_{\mathrm{c}}\right) \times F_{\mathrm{III}}\left(z_{\mathrm{c}}\right)
\end{aligned}
$$

for PopIII, and

$$
\begin{aligned}
\dot{\rho}_{\mathrm{II}}(z)= & \int_{z}^{\infty} \mathrm{d} z_{\mathrm{c}} \int_{M_{\mathrm{low}}}^{\infty} \mathrm{d} M^{\prime} N\left(M^{\prime}, z, z_{\mathrm{c}}\right) \\
& \times \dot{M}_{\mathrm{SF}}\left(M^{\prime}, z, z_{\mathrm{c}}\right) \times\left(1-F_{\mathrm{III}}\left(z_{\mathrm{c}}\right)\right)
\end{aligned}
$$

for PopII/I.

\section{Mechanical feedback processes}

\subsection{PoplI/I SNe feedback}

Mechanical feedback is associated with mechanical energy injection from $\mathrm{SNe}$ explosions and galactic winds. Most of works concentrate on the effects of the first-generation $\mathrm{SNe}$ explosions at very high redshift rather than the winds from metal-free stars (Mori et al. 2002; Yoshida et al. 2003; Salvaterra et al. 2003; Kitayama \& Yoshida 2005; Greif et al. 2007). A consequence of SNe explosions is to expel the gas out of the host halo partially (blowout) or totally (blowaway) and reduce or empty the reservoir for subsequent star formation. Some numerical simulations show that one PopIII PISN $M_{*}=200 M_{\odot}\left(E_{\mathrm{SN}} \sim 10^{52} \mathrm{erg}\right)$ can deplete its host halo easily (discussed in the next subsection). But there are at least two reasons for an SN explosion of PopII/I not being capable of blowing the most gas out of its host halo: (i) its explosion energy is much lower than that of a PopIII star, and the disperse distribution of the lifetime of PopII/I stars cannot ensure enough number of the $\mathrm{SNe}$ per unit time to deplete the halo; (ii) a halo with virial temperature $T_{\text {vir }} \geq 10^{4} \mathrm{~K}$ in the PopII/I epoch $(z \leq 14)$ having mass of $M_{\text {vir }} \simeq 10^{8} M_{\odot}$, corresponding to a binding energy $E_{\mathrm{b}} \simeq 10^{52} \sim 10^{53} \mathrm{erg}$, is able to prevent an SN explosion of PopII/I (generally $E_{\mathrm{SN}} \leq 10^{51} \mathrm{erg}$ ) from expelling most gas out of the halo, because for halos with mass $M_{\mathrm{vir}} \geq 10^{7} M_{\odot}$ the star formation will not be quenched even if $E_{\mathrm{SN}}$ exceeds the binding energy of halos $E_{\mathrm{b}}$ by 2 orders of magnitude (Kitayama \& Yoshida 2005). Furthermore, the enriched metallicity can enhance the gas cooling, which can also help to hold the escaping gas back.

However, the huge energy generated by a (PopII/I) SN explosion can, at least, partially heat the cold gas even in a 
high-metallicity environment. This normal SNe feedback has been extensively studied both in terms of SNe explosions and galactic outflows. Due to the feedback of supernovae explosions, the gas will be removed from the cold phase at the rate (Granato et al. 2004):

$$
\begin{aligned}
\dot{M}_{\mathrm{II}}^{\mathrm{SN}} & =\frac{2}{3} \dot{M}_{\mathrm{SF}}\left(M, z, z_{\mathrm{c}}\right) \epsilon_{\mathrm{SN}}^{\mathrm{II}} \frac{\eta_{\mathrm{SN}}^{\mathrm{II}} E_{\mathrm{SN}}^{\mathrm{II}}}{\sigma^{2}} \\
& =1.1 \epsilon_{\mathrm{SN}}^{\mathrm{II}} \dot{M}_{\mathrm{SF}}\left(M, z, z_{\mathrm{c}}\right)\left(\frac{500 \mathrm{~km} \mathrm{~s}^{-1}}{V_{\mathrm{c}}}\right)^{2},
\end{aligned}
$$

where $\eta_{\mathrm{SN}}^{\mathrm{II}} \simeq 6 \times 10^{-3} M_{\odot}^{-1}$ is the number of $\mathrm{SNe}$ expected per solar mass of formed stars, $E_{\mathrm{SN}}^{\mathrm{II}}$ is the kinetic energy of the ejecta from each PopII/I supernova $\left(6 \times 10^{50} \mathrm{erg}\right.$; e.g. Nadyozhin $2003)$, and $\epsilon_{\mathrm{SN}}^{\mathrm{II}}$ is the fraction of this energy that is used to heat the cold gas. Here $\eta_{\mathrm{SN}}^{\mathrm{II}}$ is evaluated by "Starburst99" and adopting a minimum progenitor mass of $8 M_{\odot}$ and the Salpeter IMF. Some analyses show that above $90 \%$ of the SN kinetic energy may be lost by radiative cooling (Thornton et al. 1998; Heckman et al. 2000). Here we set $\epsilon_{\mathrm{SN}}^{\mathrm{II}}=0.02$. As the line-of-sight velocity dispersion $\sigma$, we adopt the relationship $\sigma \simeq 0.65 V_{\text {c }}$ (Ferrarese 2002). Finally, we can obtain the SNe feedback on PopII/I SFRD:

$$
\begin{aligned}
\dot{\rho}_{\mathrm{II}}^{\mathrm{FB}}(z)= & \int_{z}^{\infty} \mathrm{d} z_{\mathrm{c}} \int_{M_{\mathrm{low}}}^{\infty} \mathrm{d} M^{\prime} N\left(M^{\prime}, z, z_{\mathrm{c}}\right) \\
& \times\left[1-F_{\mathrm{III}}\left(z_{\mathrm{c}}\right)\right] \times \dot{M}_{\mathrm{SF}}^{\mathrm{II}}\left(M^{\prime}, z, z_{\mathrm{c}}\right),
\end{aligned}
$$

where $\dot{M}_{\mathrm{SF}}^{\mathrm{II}}\left(M, z, z_{\mathrm{c}}\right)$ is the regulated SFR by the feedback from SNe explosions in PopII/I dominated halos (with $T_{\text {vir }} \geq 10^{4} \mathrm{~K}$, see Appendix A for detail).

\subsection{Mechanical feedback from first-generation stars}

The ultimate fate of a metal-free star depends critically on its mass (Heger \& Woosley 2002; Heger et al. 2003): (1) $8 M_{\odot}<$ $M_{*}<25 M_{\odot}$ (these stars explode as core-collapse $\mathrm{SNe}$ and leave neutron stars behind); (2) $25 M_{\odot}<M_{*}<40 M_{\odot}$ (these explode as faint type II SNe and leave black holes behind); (3) $40 M_{\odot}<$ $M_{*}<140 M_{\odot}$ (these do not explode as $\mathrm{SNe}$ and directly collapse into black holes ${ }^{2}$ ); (4) $140 M_{\odot}<M_{*}<260 M_{\odot}$ (these explode as PISNe, causing complete disruption); (5) $260 M_{\odot}<M_{*}$ (these collapse, in the absence of rotation, directly into black holes), so not all the PopIII stars can die as an SN explosion. We employ a slightly top-heavy IMF (Larson 1998) for PopIII stars $\left(50 M_{\odot} \leq\right.$ $\left.M_{*} \leq 500 M_{\odot}\right)$ :

$$
\frac{\mathrm{d} N}{\left(\mathrm{~d} \log M_{*}\right)} \propto\left(1+M_{*} / M_{\mathrm{c}}\right)^{-1.35},
$$

where $M_{\mathrm{c}}=100 M_{\odot}$ is the characteristic stellar mass of PopIII. As a result, the PISN is almost the only type of supernova explosion for PopIII. Bromm et al. (2003) show that, for a halo of mass $M \sim 10^{6} M_{\odot}$ at $z \sim 20$, a PISN of mass $M_{*}=250 M_{\odot}$ $\left(E_{\mathrm{SN}} \sim 10^{53} \mathrm{erg}\right)$ can disrupt the halo completely. A similar result has been obtained by Greif et al. (2007), who find that a PISN with mass $M_{*}=200 M_{\odot}\left(E_{\mathrm{SN}} \sim 10^{52} \mathrm{erg}\right)$ can deplete the whole host halo.

\footnotetext{
${ }^{2}$ But some of them experience a pulsating instability and eject their outer envelope, again leaving black holes behind.
}

The photoevaporation effect might be particularly important for PopIII objects (Ciardi \& Ferrara 2005). In small-halo objects $\left(T_{\text {vir }}<10^{4} \mathrm{~K}\right)$, photoevaporation alone from OB stars can produce strong galactic winds that expel most of the gas from galaxies. Galactic winds produced by an $\mathrm{SN}$ explosion may be important after about 10 Myr (Ricotti et al. 2008). Because the internal photoevaporation in a PopIII object is able to deplete most of the gas before the first SNe explode, in fact, the first $\mathrm{SNe}$ explosions have no chance to exert negative feedback on the star formation in these halos. Finally we only consider the SNe feedback in halos of $T_{\text {vir }}>10^{4} \mathrm{~K}$ in this paper. For those big halos $\left(T_{\text {vir }}>10^{4} \mathrm{~K}\right)$, the high cooling effect and deeply gravitational well can confine the gas photoevaporation. And even the most powerful PISN ( $E_{\mathrm{SN}} \simeq 10^{53} \mathrm{erg}$ ) cannot significantly blow the gas away (Kitayama \& Yoshida 2005). In reference to the mechanism of the SNe feedback of PopII/I, we use the same formula but with different parameters to describe the PopIII-SNe feedback in these halos $\left(M>10^{8} M_{\odot}\right)$,

$$
\begin{aligned}
\dot{M}_{\mathrm{III}}^{\mathrm{SN}} & =\frac{2}{3} \dot{M}_{\mathrm{SF}}\left(M, z, z_{\mathrm{c}}\right) \epsilon_{\mathrm{SN}}^{\mathrm{III}} \frac{\eta_{\mathrm{SN}}^{\mathrm{III}} E_{\mathrm{SN}}^{\mathrm{III}}}{\sigma^{2}} \\
& =4.5 \epsilon_{\mathrm{SN}}^{\mathrm{III}} \dot{M}_{\mathrm{SF}}\left(M, z, z_{\mathrm{c}}\right)\left(\frac{500 \mathrm{~km} \mathrm{~s}^{-1}}{V_{\mathrm{c}}}\right)^{2}
\end{aligned}
$$

Here $E_{\mathrm{SN}}^{\mathrm{III}} \simeq 10^{52} \mathrm{erg}, \eta_{\mathrm{SN}}^{\mathrm{III}} \simeq 1.5 \times 10^{-3} M_{\odot}^{-1}$ (evaluated with the PopIII IMF and mass range). For lack of information about the strength factor of the SNe feedback of PopIII, we set $\epsilon_{\mathrm{SN}}^{\mathrm{III}}=0.05$ (Granato et al. 2004; Lapi et al. 2006) as our reference value. One can obtain the PopIII SFRD with SNe feedback:

$$
\begin{aligned}
\dot{\rho}_{\mathrm{III}}^{\mathrm{FB}}(z)= & \int_{z}^{\infty} \mathrm{d} z_{\mathrm{c}} F_{\mathrm{III}}\left(z_{\mathrm{c}}\right) \int_{M\left(z_{\mathrm{c}}, 10^{4} \mathrm{~K}\right)}^{\infty} N\left(M^{\prime}, z, z_{\mathrm{c}}\right) \\
& \times \dot{M}_{\mathrm{SF}}^{\mathrm{III}}\left(M^{\prime}, z, z_{\mathrm{c}}\right) \mathrm{d} M^{\prime}
\end{aligned}
$$

where $\dot{M}_{\mathrm{SF}}^{\mathrm{III}}\left(M, z, z_{\mathrm{c}}\right)$ is the regulated SFR by the feedback from SNe explosions in PopIII halos of $T_{\text {vir }}>10^{4} \mathrm{~K}$ (see Appendix A for detail). Of course, it is definite that the number density of halos with $T_{\text {vir }}>10^{4} \mathrm{~K}$ is tiny in PopIII epoch according to the hierarchical clustering scenario.

Figure 1 shows the SNe feedback in PopIII $\left(T_{\text {vir }}>10^{4} \mathrm{~K}\right)$ and PopII/I halos. In the left panel, we scale down all the observation data by a factor 1.9 to make it consistent with the IMF used in our model $\left(M_{*}^{\min }=0.5 M_{\odot}, M_{*}^{\max }=100 M_{\odot}\right.$ for PopII/I stars and Salpeter type IMF). In the right panel, the original SFRD of PopIII $\left(T_{\text {vir }}>10^{4} \mathrm{~K}\right)$ is cut down about one order of magnitude by the SNe feedback.

Figure 1 manifests the relative feedback strength of SNe feedback $\left[\dot{\rho}_{\mathrm{III}}^{\mathrm{FB}}(z) / \dot{\rho}_{\mathrm{III}}(z)\right.$ or $\left.\dot{\rho}_{\mathrm{II} / \mathrm{I}}^{\mathrm{FB}}(z) / \dot{\rho}_{\mathrm{II} / \mathrm{I}}(z)\right]$ reduces as the redshift decreases, because massive halos appear abundant only at low redshift according to the "bottom-up" hierarchical structure model (see Mo \& White 2002, for details) and massive halos whose binding energy is much higher than before can reduce the feedback effect from SNe explosions. On the other hand, massive stars disappear as the metallicity of gas is enriched. Small star formation with a long lifetime and small $E_{\mathrm{SN}}$ is enhanced in the metal-enriched cloud.

\section{Radiative feedback and cosmic reionization}

The ionizing radiation produced by massive stars or quasars can have local effects or long-range effects, either affecting the formation and evolution of nearby objects or joining the radiation 

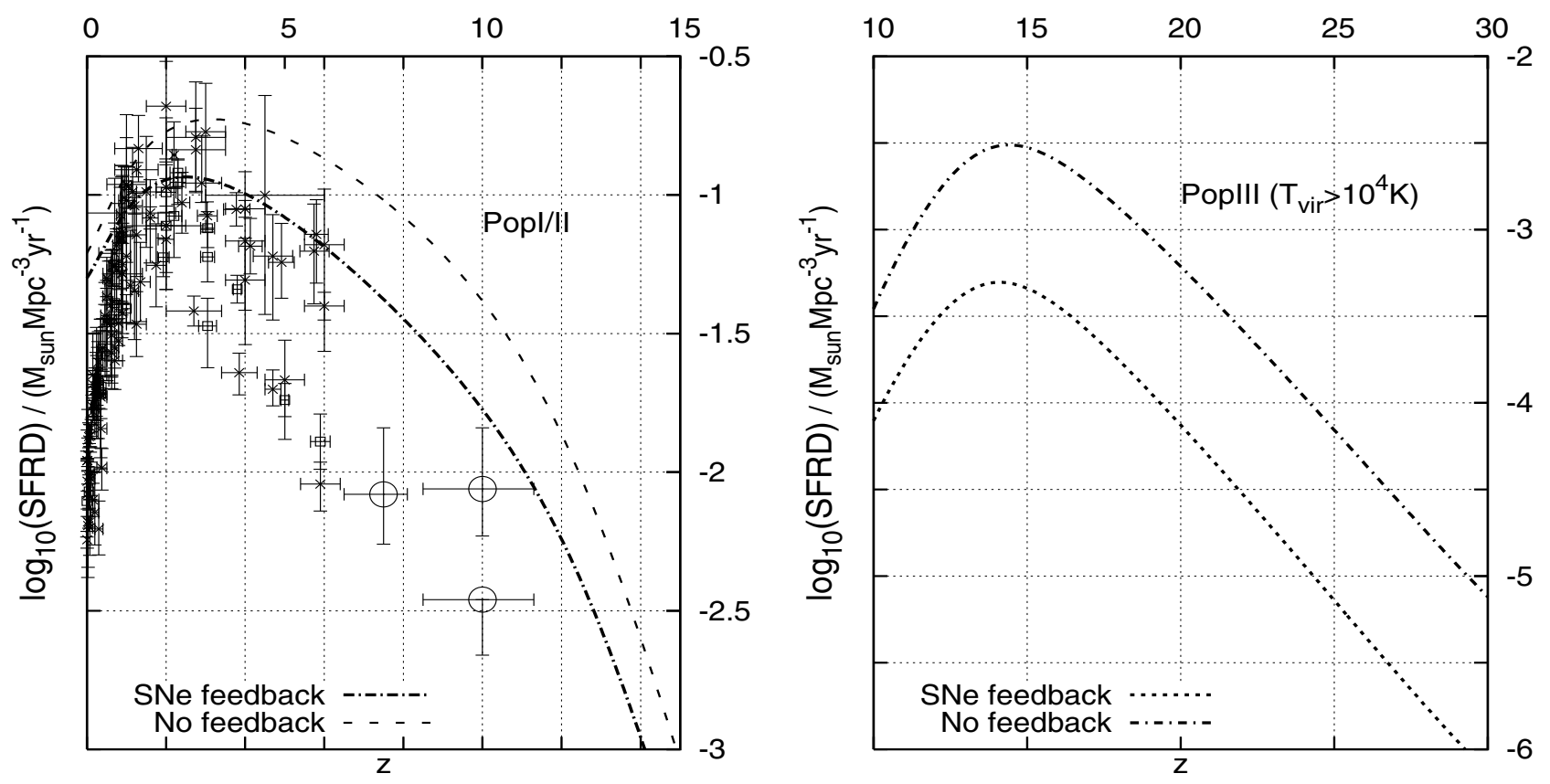

Fig. 1. Evolution of SFRD with the redshift z. Left panel: the crossed data points are taken from Hopkins \& Beacom (2006). The squared ones come from Reddy et al. (2008). Three circled points are from the UDF data (Bouwens et al. 2005). The dashed line represents the PopII/I SFRD without SNe feedback, while the dot-dashed one with the SNe feedback. Right panel: the original SFRD of PopIII $\left(T_{\text {vir }}>10^{4} \mathrm{~K}\right.$, the dot-dashed line) is suppressed down about one order of magnitude by the SNe feedback (the dotted line).

produced by other galaxies to form a background. Mini-QSOs that are usually thought to be powered by accretion onto the first black holes may provide an X-ray background when the universe has a certain number of mini-QSOs in a unit comoving volume. This background would ionize the $\mathrm{HI}$ to $\mathrm{H}^{+}$and $\mathrm{e}^{-}$by direct photoionization or collisional excitation from a high-energy photoelectron produced by photoionization. If this event occurs at the core of a protogalaxy, the fraction of molecular hydrogen would be promoted (Haiman et al. 2000; Glover \& Brand 2003) via

$\mathrm{H}+\mathrm{e}^{-} \rightarrow \mathrm{H}^{-}+\gamma$

$\mathrm{H}^{-}+\mathrm{H} \rightarrow \mathrm{H}_{2}+\mathrm{e}^{-}$.

Dijkstra et al. (2004) and Salvaterra et al. (2005) show that the hard X-ray from the same sources will produce a present-day soft X-ray background. They find that the models with accreting black holes will overproduce the observed X-ray background by a large factor. A population dominated by mini-QSOs could still partially ionize the IGM at $z>6$, but its contribution can be severely constrained if the X-ray background is resolved further into discrete sources. By considering the constraint from the soft X-ray background, Ricotti \& Ostriker (2004b) show that miniquasars at high- $z$ do not overproduce the X-ray background and can still produce a significant contribution to reionization and the optical depth of electrons $\tau_{\mathrm{e}}$.

Based on the investigation of the environment and reionization process around the highest redshift QSOs having Gunn-Peterson troughs $(z>6.1)$, Yu \& Lu (2005) argue that a significant fraction of hydrogen in the Stromgren sphere around QSOs is ionized by photons from stars and that only about several percent to at most 10-20\% of the total hydrogen is left (e.g., in minihalos, halos, or high-density subregions) to be ionized by QSO photons. Willott et al. (2005) show that the current constraints on the quasar population give an ionizing photon density $\ll 30 \%$ that of the star-forming galaxy population by analyzing the observational data from the Canada-France-Hawaii
Telescope. They conclude that active galactic nuclei make a negligible contribution to the reionization of hydrogen at $z \sim 6$. But this argument only applies to quasars at $z \leq 6$, one can still have a high- $z$ population of mini-quasars that partially ionize the IGM at $z>10$ without overproducing the X-ray background (Ricotti et al. 2005).

Pre-ionization by X-rays can increase the IGM optical depth from $\tau_{\mathrm{e}} \simeq 0.06$ given by stellar sources only to $0.1 \leq \tau_{\mathrm{e}} \leq 0.2$ (Ricotti \& Ostriker 2004b). From $4 \sim 5$ years ago, it was necessary to fit an observational value of $\tau_{\mathrm{e}} \simeq 0.17$ measured by WMAP satellite. Recent WMAP data indicating $0.068 \leq \tau_{\mathrm{e}} \leq$ 0.1 maybe imply that pre-ionization by $\mathrm{X}$-rays is not as important as before. In this paper, we focus on the ionization by stellar sources and ignore the effects from QSOs or mini-QSOs. We will consider the AGN feedback carefully in the work in preparation.

\subsection{Photoevaporation in small-halo objects $\left(T_{\text {vir }}<10^{4} \mathrm{~K}\right)$}

PopIII stars quite likely reside in small DM halos and a metalfree environment. It benefits the photons escaping and the radiative cooling. As mentioned in the last section, photoevaporation is important in small-halo objects $\left(T_{\text {vir }}<10^{4} \mathrm{~K}\right.$, e.g. most of the PopIII halos). It can prevent the size of HII regions from exceeding $R_{\mathrm{HII}}^{\mathrm{com}} \simeq 5 h^{-1} \mathrm{kpc}$, the mean free path of ionizing photons, about the size of the dense filaments and the virial radii of the halos (Ricotti et al. 2002b). When the HII regions become bigger than the filaments, molecular hydrogen is destroyed and the star formation is suppressed. An analytic model has been developed to describe the propagation of ionization fronts in the IGM (Barkana \& Loeb 2001):

$\left\langle n_{\mathrm{ISM}}^{\mathrm{H}}\right\rangle \frac{\mathrm{d} V_{\mathrm{p}}}{\mathrm{d} t}=\frac{\mathrm{d} N_{\gamma}}{\mathrm{d} t}-\alpha_{\mathrm{B}}\left\langle\left(n_{\mathrm{ISM}}^{\mathrm{H}}\right)^{2}\right\rangle V_{\mathrm{p}}$,

where $N_{\gamma}$ is the number of ionizing photons produced by the source. Here $V_{\mathrm{p}}$ is the ionized proper volume, $\alpha_{\mathrm{B}} \simeq$ $2.6 \times 10^{-13} \mathrm{~cm}^{3} \mathrm{~s}^{-1}$ is the case $\mathrm{B}$ recombination coefficient 
at $T \simeq 10^{4} \mathrm{~K}$ (Seager et al. 1999; Barkana \& Loeb 2001), and $n_{\mathrm{ISM}}^{\mathrm{H}}$ is the proper number density of the hydrogen atoms. We assume the ISM in halos is homogeneous, which means $\left\langle\left(n_{\mathrm{ISM}}^{\mathrm{H}}\right)^{2}\right\rangle=\left\langle\left(n_{\mathrm{ISM}}^{\mathrm{H}}\right)\right\rangle^{2}$, so the analytical solution is

$$
\begin{aligned}
V_{\mathrm{p}}\left(t, t\left(z_{\mathrm{c}}\right)\right)= & \int_{t\left(z_{\mathrm{c}}\right)}^{t} \frac{1}{\left\langle n_{\mathrm{ISM}}^{\mathrm{H}}\left(t^{\prime}\right)\right\rangle} \frac{\mathrm{d} N_{\gamma}}{\mathrm{d} t^{\prime}} \\
& \times \exp \left\{-\alpha_{\mathrm{B}} \int_{t^{\prime}}^{t}\left\langle n_{\mathrm{ISM}}^{\mathrm{H}}\left(t^{\prime \prime}\right)\right\rangle \mathrm{d} t^{\prime \prime}\right\} \mathrm{d} t^{\prime},
\end{aligned}
$$

where $^{3}$

$\left\langle n_{\mathrm{ISM}}^{\mathrm{H}}(t)\right\rangle=\Delta_{\mathrm{c}}(t) \times \frac{\Omega_{\mathrm{b}}}{\Omega_{\mathrm{m}}} \times \bar{n}_{\mathrm{H}}^{0} \times(1+z(t))^{3}$,

$\bar{n}_{\mathrm{H}}^{0}=1.88 \times 10^{-7}\left(\frac{\Omega_{b} h^{2}}{0.022}\right) \mathrm{cm}^{-3}$,

and $\bar{n}_{\mathrm{H}}^{0}$ is the present number density of hydrogen. As mentioned in Eq. (3), $\Delta_{\mathrm{c}}$ is the overdensity relative to the critical density at the collapse redshift. Following Barkana \& Loeb (2001), we can evaluate $\mathrm{d} N_{\gamma} / \mathrm{d} t$ by using

$\frac{\mathrm{d} N_{\gamma}}{\mathrm{d} t}=\frac{\alpha-1}{\alpha} \frac{n_{\gamma}^{\mathrm{III}}}{t_{\mathrm{s}}}$,

where $\alpha$ is the index in the mass-luminosity relation (for OB stars $\alpha=4.5$ ), and $n_{\gamma}^{\text {III }}$ the number of ionizing photons released per baryon of stars formed (Schaerer 2003; Haiman \& Bryan 2006). Fang \& Cen (2004) present a relationship between $n_{\gamma}^{\mathrm{III}}$ and the stellar mass, $M_{*}$. Because $n_{\gamma}^{\mathrm{III}}$ is not sensitive to $M_{*}$ in the mass range of concern, we set $n_{\gamma}^{\text {III }}$ as a fixed value 80000 in this paper (Fang \& Cen 2004). Most massive stars fade away with the characteristic time scale $t_{\mathrm{s}}=3 \times 10^{6} \mathrm{yr}$ (Bond et al. 1984; Alvarez et al. 2006). For a halo of mass $M$, star formation initiated at $t\left(z_{\mathrm{c}}\right)$, the proper size (or radii) of the HII region at later time $t$, is

$R\left(M, t\left(z_{\mathrm{c}}\right), t\right)=\left\{\frac{3}{4 \pi} f_{*}^{\mathrm{III}} f_{\mathrm{esc}}^{\mathrm{III}} \frac{\Omega_{\mathrm{b}} M}{\Omega_{\mathrm{m}} m_{\mathrm{p}}} \times V_{\mathrm{p}}\left(t, t\left(z_{\mathrm{c}}\right)\right)\right\}^{1 / 3}$,

where $f_{\text {esc }}^{\mathrm{III}}$ is the escape fraction of ionizing photons from the sources (or resolution elements in 3D numerical simulations, see Ricotti et al. 2002a,b, 2008), and $f_{\text {esc }}^{\mathrm{III}} \simeq 0.3 \sim 0.9$ is suggested by Choudhury \& Ferrara (2006) and Mao et al. (2007). Yoshida et al. (2007) provide a time-averaged ionizing photon escape fraction as a function of stellar mass $M_{*}$. For the mass range adopted in this work, we use a fixed value $f_{\text {esc }}^{\text {III }}=0.8$. As mentioned above, star formation will be suppressed when $R\left(M, t^{\prime}\left(z_{\mathrm{c}}\right), t^{\prime}\right)$ is equal to the size of the filaments. Thus the relation between $t^{\prime}-t^{\prime}\left(z_{\mathrm{c}}\right)$ and $M$ is given by

$R\left(M, t^{\prime}\left(z_{\mathrm{c}}\right), t^{\prime}\right)=R_{\mathrm{HII}}^{\mathrm{com}} /\left(1+z^{\prime}\right)$.

We illustrate this relationship in Fig. 2, where $\tau_{\mathrm{FB}}=t^{\prime}-t^{\prime}\left(z_{\mathrm{c}}\right)$ is the duration time of star formation. We plot five cases $z_{\mathrm{c}}=$ $10,15,20,25,30$, which are actually not straight lines in the figure. For the halos with the same mass, $\tau_{\mathrm{FB}}$ increases as the redshift decreases. The bigger the halo, the less time it spends enlarging the HII region to the size of the filaments. If $\tau_{\mathrm{FB}}<t_{\mathrm{s}}$, the photoevaporation feedback occurs before the first SN exploded.

\footnotetext{
${ }^{3}$ The mass fraction of baryons in a halo is $\frac{\Omega_{\mathrm{b}}}{\Omega_{\mathrm{m}}}$, so the baryon density in halos is at least $\Delta_{\mathrm{c}}(t) \times \frac{\Omega_{\mathrm{b}}}{\Omega_{\mathrm{m}}}$ times the one in IGM.
}

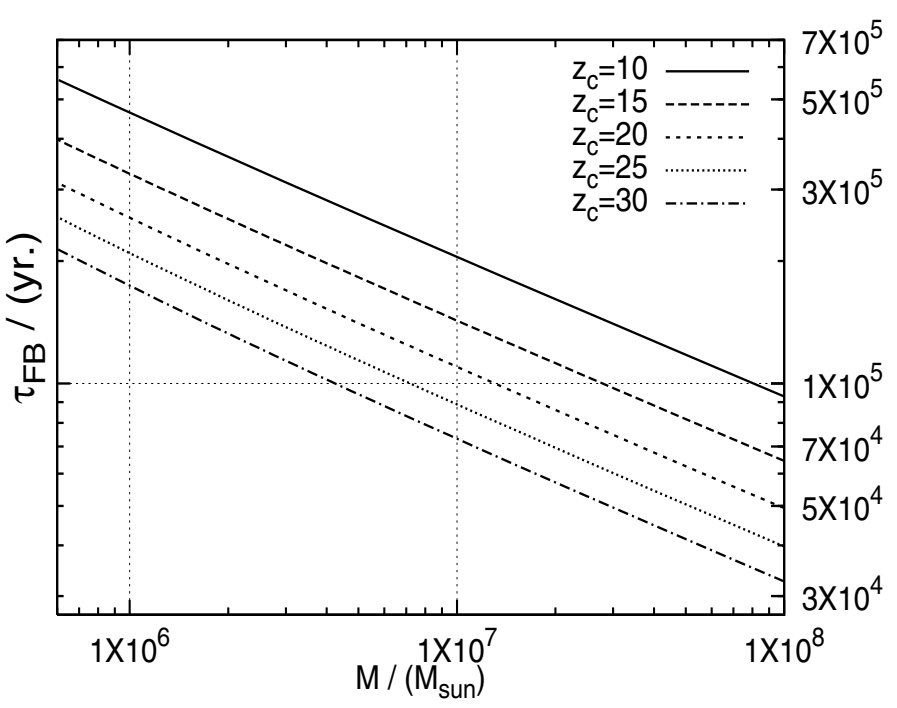

Fig. 2. The duration time of star formation $\tau_{\mathrm{FB}}=t^{\prime}-t^{\prime}\left(z_{\mathrm{c}}\right)$ versus the masses of halos.

And the inequality $c \times \tau_{\mathrm{FB}}>R_{\mathrm{HII}}^{\mathrm{com}} /\left(1+z^{\prime}\right)(c$ the speed of light $)$ is valid for all these cases, which means the speed of the propagation of the ionization fronts cannot exceed the speed of the light $^{4}$.

Now we can calculate the SFRD in PopIII halos with $T_{\text {vir }}<$ $10^{4} \mathrm{~K}$ by using

$$
\begin{aligned}
\dot{\rho}_{\mathrm{III}}^{\mathrm{FB}}\left(z,<10^{4} \mathrm{~K}\right)= & \int_{z}^{\infty} \mathrm{d} z_{\mathrm{c}} F_{\mathrm{III}}\left(z_{\mathrm{c}}\right) \int_{M\left(z_{\mathrm{c}}, 10^{3} \mathrm{~K}\right)}^{M\left(z_{\mathrm{c}}, 10^{4} \mathrm{~K}\right)} N\left(M^{\prime}, z, z_{\mathrm{c}}\right) \\
& \times \theta\left(t\left(z_{\mathrm{c}}\right)+\tau_{\mathrm{FB}}-t_{\mathrm{z}}\right) \times \dot{M}_{\mathrm{SF}}\left(M^{\prime}, z, z_{\mathrm{c}}\right) \mathrm{d} M^{\prime}
\end{aligned}
$$

where $\theta$ is a Heaviside function. Figure 3 shows the effects of this kind of feedback: $(i)$ they suppress the PopIII SFRD down about one order of magnitude at the peak $(z \sim 15)$; (ii) photoevaporation gets stronger when redshift decreases. And we also find that the feedback will become weak when $f_{\text {esc }}^{\text {III }}$ is tuned down. Moreover, the SFRD is not sensitive to the $f_{*}^{\text {III }}$.

\subsection{Radiative feedback in large halos $\left(T_{\text {vir }}>10^{4} \mathrm{~K}\right)$}

For halos with $T_{\text {vir }}$ higher than the cooling temperature of the hydrogen atoms $\left(10^{4} \mathrm{~K}\right)$, gas cools via $\mathrm{HI}$ emission lines, and the photoevaporation by internal sources cannot expel gas significantly. But the ionizing background can still suppress some small halos in which the star formation is about to initiate. Due to the IGM reionized by UV photon escape from star-forming galaxies, the temperature of the gas in halos is enhanced. This can dramatically increase the Jeans mass. Furthermore, numerical simulations indicate that the photoionizing background can completely suppress galaxy formation in halos with circular velocity $V_{\mathrm{c}} \leq 35 \mathrm{~km} \mathrm{~s}^{-1}$, while the mass of cooled baryons is reduced by $50 \%$ for halos with circular velocities $\sim 50 \mathrm{~km} \mathrm{~s}^{-1}$ (Thoul \& Weinberg 1996). In the ionized fraction of the universe, we assume complete suppression of star formation in halos below circular velocity $V_{\mathrm{c}}=35 \mathrm{~km} \mathrm{~s}^{-1}$ and no suppression above circular velocity of $95 \mathrm{~km} \mathrm{~s}^{-1}$. For intermediate masses,

\footnotetext{
${ }^{4}$ However, it seems that the offcenter sources may make a superluminal spread when their HII regions get an overlap. But it is not real superluminal behavior.
} 


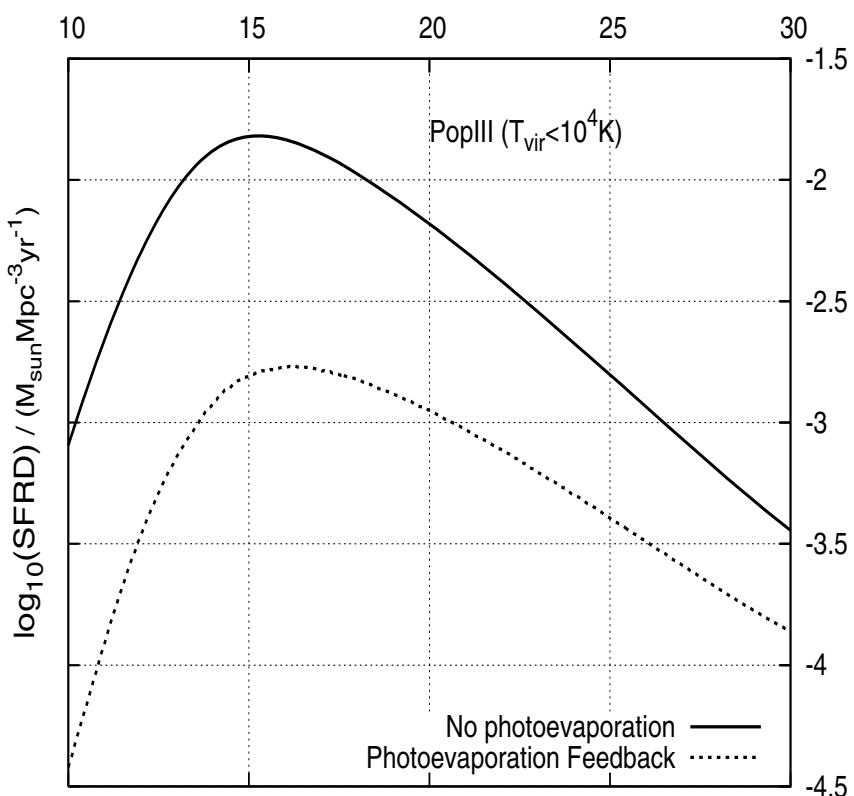

Z

Fig. 3. Evolution of SFRD as a function of the redshift $z$ with/without the photoevaporation feedback effect. The solid line represents the SFRD without feedback. The dotted one stands for the case that the SF is suppressed by photoevaporation. Both of them represent the PopIII SFRD in halos with $T_{\text {vir }}<10^{4} \mathrm{~K}$.

we adopt a linear fit from 1 to 0 for the suppression factor (as in Bromm \& Loeb 2002). Thus in the HII region the SFRD can be expressed by

$$
\begin{aligned}
\dot{\rho}_{\mathrm{II}}^{\mathrm{HII}}(z)= & \int_{z}^{\infty} \mathrm{d} z_{\mathrm{c}} \int_{M\left(z_{\mathrm{c}}, 10^{4} \mathrm{~K}\right)}^{\infty} \mathrm{d} M^{\prime} N\left(M^{\prime}, z, z_{\mathrm{c}}\right) \\
& \times\left[1-F_{\mathrm{III}}\left(z_{\mathrm{c}}\right)\right] \times \dot{M}_{\mathrm{SF}}^{\mathrm{II}}\left(M^{\prime}, z, z_{\mathrm{c}}\right) \times W\left(V_{\mathrm{c}}\right)
\end{aligned}
$$

for PopII/I and

$$
\begin{aligned}
\dot{\rho}_{\mathrm{III}}^{\mathrm{HII}}\left(z,>10^{4} \mathrm{~K}\right)= & \int_{z}^{\infty} \mathrm{d} z_{\mathrm{c}} F_{\mathrm{III}}\left(z_{\mathrm{c}}\right) \int_{M\left(z_{\mathrm{c}}, 10^{4} \mathrm{~K}\right)}^{\infty} N\left(M^{\prime}, z, z_{\mathrm{c}}\right) \\
& \times \dot{M}_{\mathrm{SF}}^{\mathrm{III}}\left(M^{\prime}, z, z_{\mathrm{c}}\right) \times W\left(V_{\mathrm{c}}\right) \mathrm{d} M^{\prime}
\end{aligned}
$$

for PopIII, where $W\left(V_{\mathrm{c}}\right)$ is

$$
W\left(V_{\mathrm{c}}\right)= \begin{cases}0, & V_{\mathrm{c}} \leq 35 \mathrm{~km} \mathrm{~s}^{-1} \\ \left(V_{\mathrm{c}}-35\right) /(95-35), & 35 \leq V_{\mathrm{c}} \leq 95 \mathrm{~km} \mathrm{~s}^{-1} \\ 1, & V_{\mathrm{c}} \geq 95 \mathrm{~km} \mathrm{~s}^{-1} .\end{cases}
$$

We plot two extreme cases in Fig. 4: the stars form in the completely reionized universe in HII region and in the completely neutral universe in $\mathrm{HI}$ region. This radiative feedback from PopII/I(left panel) and PopIII has the same behavior: feedback gets stronger when redshift increases. Similar to the SNe mechanical feedback mentioned above, the more massive the halo, the less the SF can be affected.
For the ionized fraction of the universe at a given redshift $z$, it can be described by the following equation. For simplicity, we assume that all the Lymann continuum photons escape from a star-forming galaxy are involved in reionizing the IGM, and the photons emitted from the sources will immediately join the action with the atom, regardless of the photon propagation between the source and the atom ${ }^{5}$. Then the fraction of ionized hydrogen $Q_{\mathrm{HII}}$, the so-called filling factor, evolves as (Barkana \& Loeb 2001),

$$
\frac{\mathrm{d} Q_{\mathrm{HII}}}{\mathrm{d} z}=\frac{f_{*} f_{\mathrm{esc}}}{(1+z)^{3} \bar{n}_{\mathrm{H}}^{0}} \frac{\mathrm{d} N_{\gamma}}{\mathrm{d} t} \frac{\mathrm{d} t}{\mathrm{~d} z}-\alpha_{\mathrm{B}}(1+z)^{3} \bar{n}_{\mathrm{H}}^{0} Q_{\mathrm{HII}} C \frac{\mathrm{d} t}{\mathrm{~d} z},
$$

where the volume-averaged clumping factor of the IGM, $C$, is defined as $C \equiv\left\langle n_{\mathrm{H}}^{2}\right\rangle /\left((1+z)^{3} \bar{n}_{\mathrm{H}}^{0}\right)^{2}$. The first term on the right is the rate of ionization and the second term is the rate of recombination, weighted by the $Q_{\mathrm{HII}}$, as recombinations take place only in the ionized region. Then $\mathrm{d} N_{\gamma} / \mathrm{d} t$ is obtained from the SFRD calculation as,

$$
\frac{\mathrm{d} N_{\gamma}}{\mathrm{d} t}=\frac{\dot{\rho}_{\mathrm{SF}}\left(z,>10^{4} \mathrm{~K}\right)(1+z)^{3}}{f_{*} m_{\mathrm{p}}} n_{\gamma},
$$

where $\dot{\rho}_{\mathrm{SF}}\left(z, T_{\text {vir }}>10^{4} \mathrm{~K}\right)$ is the SFRD in halos of $T_{\text {vir }}>10^{4} \mathrm{~K}$ (including all PopII/I halos and a few PopIII halos) at $z$. Because the strong photoevaporation feedback in small-halo objects can quench the SF, which stops the ionizing photons to escape to the IGM. This part of SFRD contribute little to the IGM reionization. The value of $n_{\gamma}$ depends on the IMF of the forming stars. For a Salpeter IMF (with $0.5 M_{\odot} \leq M_{*} \leq 100 M_{\odot}$ ), $n_{\gamma}^{\mathrm{II}}$ is about 5400, evaluated by "Starburst99" 6 with metallicity $Z=0.008$, however, for the metal-free stars, the IMF could be biased towards very massive stars. As mentioned in the last section, we still used $n_{\gamma}^{\text {III }} \sim 80000$ as our reference value. For the clumping factor of IGM, $C$, we adopted the simple form given by Haiman \& Bryan (2006).

By solving Eq. (25) (see Appendix B for detail), one can find that SF and reionization affect each other. When star formation gets high, HI ionization becomes strong and star formation is suppressed. Hopefully, JWS T can help us to understand their relationship (SF and reionization) exactly. The result is plotted in Fig. 5 (left panel). One may find that the solid line separates from the SFRD(PopII/I+PopIII) in HI region and eventually merges into the SFRD line in HII region as the filling factor $Q_{\mathrm{HII}}$ (see Fig. 6) increases from zero to unit. The PopIII SFRD in halos of $T_{\text {vir }}<10^{4} \mathrm{~K}$ is taken into account in the righthand panel in Fig. 5. By comparing these two panel, we can find that the main part of PopIII SFRD is still contributed by those stars in smallhalo objects. Since the PopIII PISNe contaminate the IGM with heavy elements at very high efficiency (recall the ultimate fate of metal-free stars), PopIII ends its era by itself quickly (survival time $\sim 2 \times 10^{8} \mathrm{yr}$ ). The ignition of PopII stars makes the reionization continue and eventually completes it at $z \sim 6$.

The reionization history and the optical depth of electrons are plotted in Fig. 6. Here we take the singly ionized HeII into account in righthand panel in Fig. 6. The equation for calculating the optical depth of electrons is:

$\tau_{\mathrm{e}}(z)=\int n_{\mathrm{e}}(z) \sigma_{\mathrm{e}} \frac{\mathrm{d} s}{\mathrm{~d} z} \mathrm{~d} z$

\footnotetext{
${ }^{5}$ Recent work shows that the finite speed of light will lead to a substantial change of the growth rate in the ionized volume (see Qiu et al. 2008 , for more details).

${ }^{6}$ http://www.stsci.edu/science/starburst99/
} 

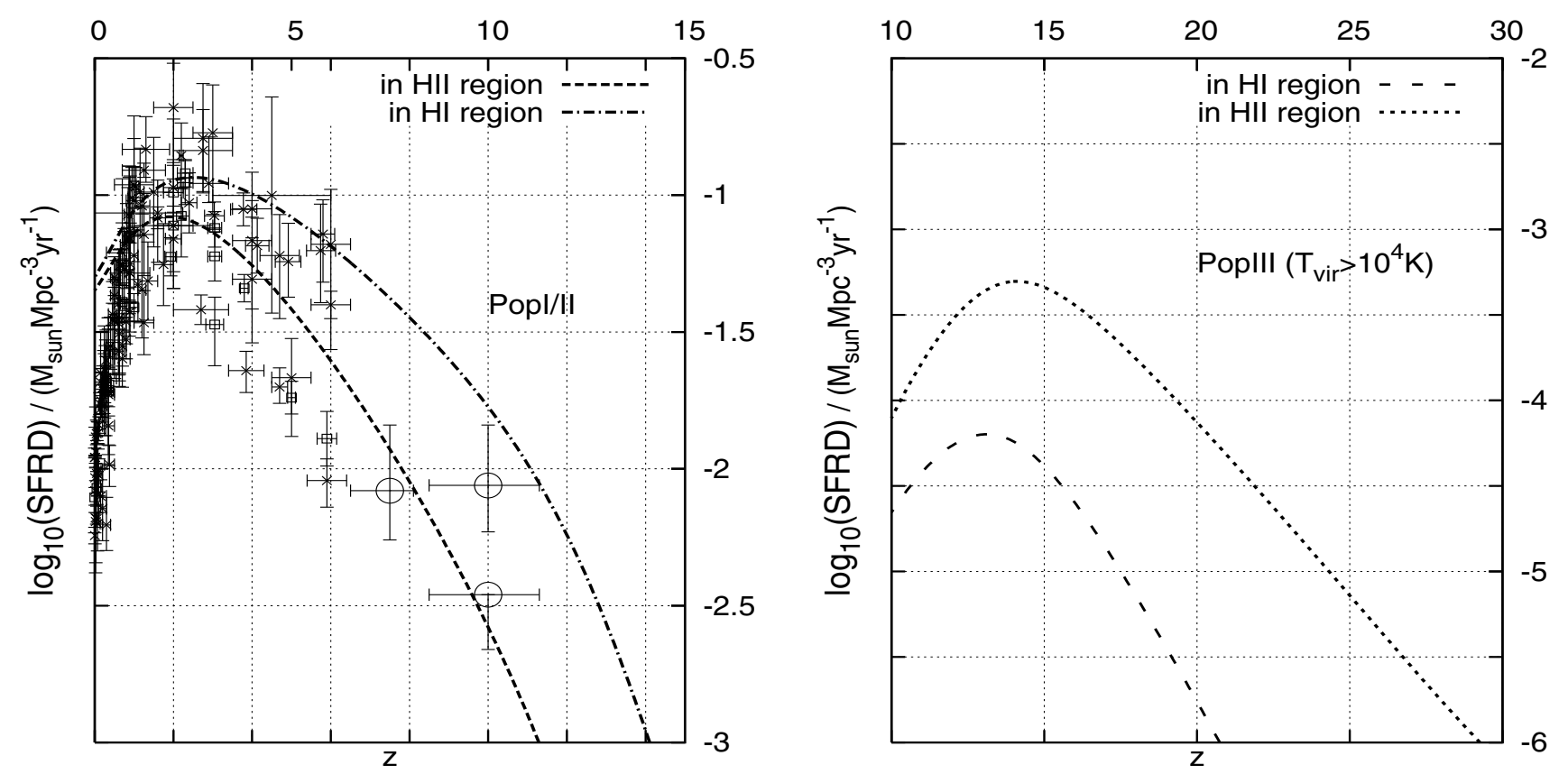

Fig. 4. Evolution of SFRD in two extreme cases: the stars form in the completely reionized universe (HII region, the dashed line) and in the completely neutral universe (HI region, the dot-dashed line in the lefthand panel and the dotted line in the right one). Observational data are the same as Fig. 1.

where $n_{\mathrm{e}}(z)$ is the mean electron number density in the universe, $\sigma_{\mathrm{e}}$ stands for the Thompson cross section for electron scattering, and $\mathrm{d} s=-c \mathrm{~d} z / H(z)(1+z)$ is the proper line element. Figure 6 illustrates that the model output can fit the observation from the highest QSOs $\left(z_{\text {re }} \sim 6\right)$, and WMAP 5 years result $\left(\tau_{\mathrm{e}}=0.084_{-0.016}^{+0.016}\right.$ Komatsu et al. 2008) at the same time. Most of the UV photons emitted from small halos that have the overwhelming majority in PopIII objects are confined in $R_{\mathrm{HII}}^{\mathrm{com}}$. As shown in the lefthand panel in Fig. 6, PopIII stars are not able to reionize the universe significantly (about $20 \%$ at $z \sim 14$ ). In the righthand panel, the optical depth to Thomson scattering provided by PopIII stars is less than $22 \%$.

\section{Discussion and conclusions}

In this paper we develop an analytic model of the mechanical and radiative feedback of star formation based on some results of numerical simulations. Basically, our results can fit the observational data. However, this model also depends on some initial parameters (most of them are listed in Table 1) and working assumptions.

(i) It is possible to fit the reionization result from the highest QSOs observation by providing another pair of $f_{*}^{\mathrm{II}}$ and $f_{\mathrm{esc}}^{\mathrm{II}}$. This is because the IGM reionization mainly depends on $n_{\gamma}^{\text {II }}$ and $f_{*}^{\mathrm{II}} \times f_{\mathrm{esc}}^{\mathrm{II}}$. The value of $n_{\gamma}^{\mathrm{II}}$ is nearly fixed under certain conditions (e.g. metallicity, IMF, and stellar mass range), but lacking the SFRD observational data constraint on $f_{*}^{\mathrm{II}}$, one can find many pairs of $f_{*}^{\mathrm{II}}$ and $f_{\text {esc }}^{\mathrm{II}}$ by keeping $f_{*}^{\mathrm{II}} \times f_{\mathrm{esc}}^{\mathrm{II}}$ invariant to guarantee $\tau_{\mathrm{e}}=0.084_{-0.016}^{+0.016}$.

(ii) As for the assumption on $F_{\text {III }}(z)$, there are a lot of similar functions. This is an uncertainty factor in this model. Hopefully, it may be determined by the numerical simulations and even the future JWST observation.

(iii) We only consider the negative feedback in this work, the neglect of some positive feedback, such as the X-ray background or dense shell (from $\mathrm{SNe}$ explosions) fragmentation, results in the underestimation of the SFRD, $Q_{\mathrm{HII}}, \tau_{\mathrm{e}}$, and so on. This is what we will investigate in the next work.

(iv) Although we can fit the optical depth to Thomson scattering in this work, it does not mean the X-ray pre-ionization is not important. Obviously, our results are close to the low fringe of the error bar. Perhaps, the left space in the error bar is waiting for the contribution from X-ray pre-ionization.

In the following, we summarize the results of this work in more detail.

1. The $\mathrm{SNe}$ feedback can suppress the star formation in large halos $\left(T_{\text {vir }}>10^{4} \mathrm{~K}\right)$. And the relative feedback strength of $\mathrm{SNe}$ feedback $\left[\dot{\rho}_{\mathrm{III}}^{\mathrm{FB}}(z) / \dot{\rho}_{\mathrm{III}}(z)\right.$ or $\left.\dot{\rho}_{\mathrm{II} / \mathrm{I}}^{\mathrm{FB}}(z) / \dot{\rho}_{\mathrm{II} / \mathrm{I}}(z)\right]$ reduces as the redshift decreases, because massive halos appear abundant at low redshift and massive halos whose binding energy is much higher than before can reduce the feedback effect from $\mathrm{SNe}$ (see Eqs. (8) and (11)).

2. The radiative feedback mechanism can affect the SFR in both small $\left(T_{\text {vir }}<10^{4} \mathrm{~K}\right)$ and $\operatorname{large}\left(T_{\text {vir }}>10^{4} \mathrm{~K}\right)$ PopIII halos. In small halos, the shallow gravitational well and the poor cooling-efficiency cannot prevent the hot gas from escaping due to the strong photoevaporation from the massive OB stars. The radiative feedback can suppress the PopIII SFR considerably. In large halos, the ionizing background enhances the Jeans mass and makes the SFR suppressed more or less.

3. The SFRD in small PopIII halos $\left(T_{\text {vir }}<10^{4} \mathrm{~K}\right)$ is sensitive to $\tau_{\mathrm{FB}}$, which depends on $f_{\mathrm{esc}}^{\mathrm{III}}$ and $n_{\gamma}^{\mathrm{III}}$ seriously. If $f_{\mathrm{esc}}^{\mathrm{III}}$ and $n_{\gamma}^{\mathrm{III}}$ increases, $\tau_{\mathrm{FB}}$ will decreases and so does the SFRD. Furthermore, our results support this viewpoint: early star formation is likely to be self-regulated (Ricotti et al. 2002a,b; Yoshida et al. 2003; Ciardi \& Ferrara 2005; Kitayama \& Yoshida 2005; Ricotti et al. 2008). 

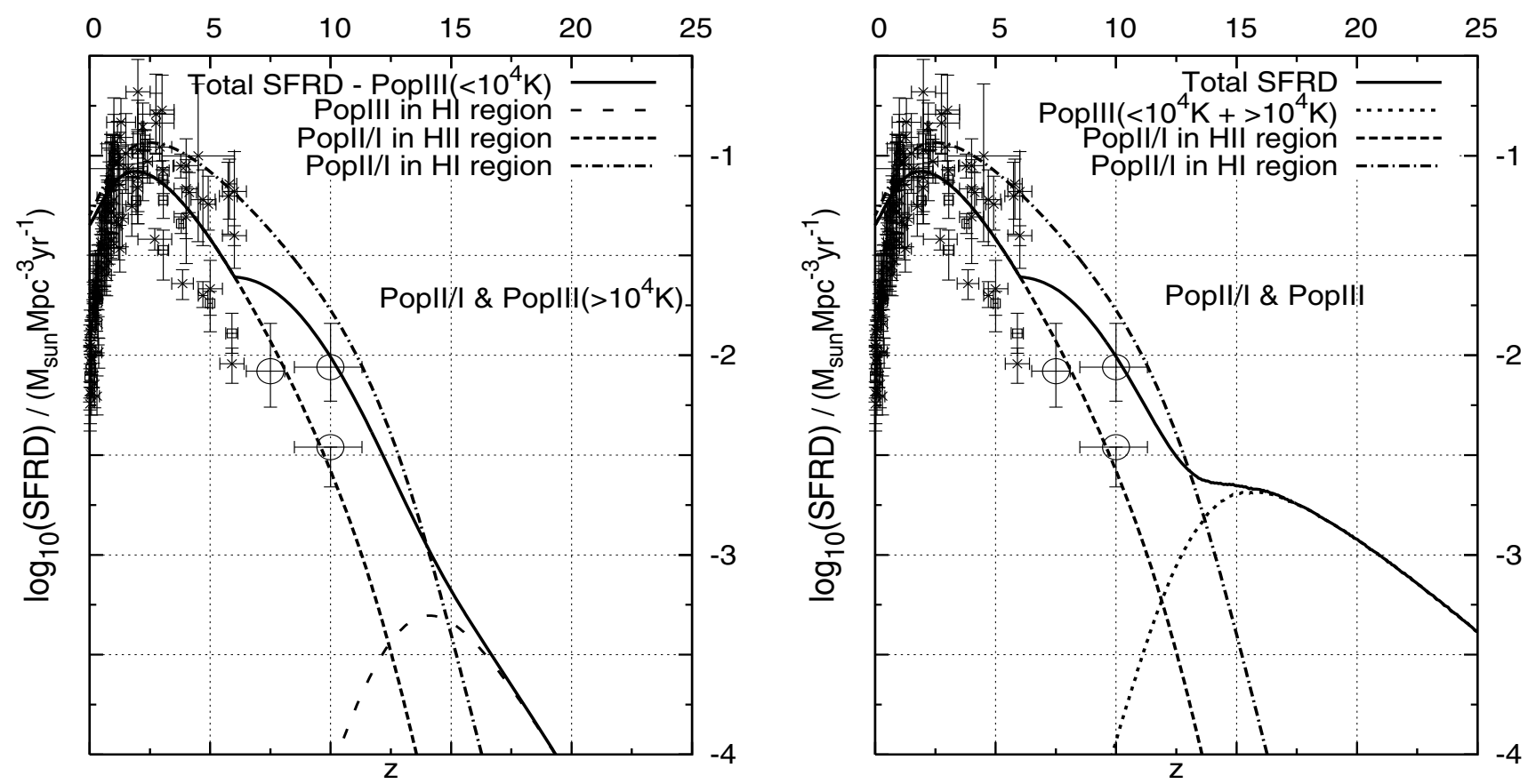

Fig. 5. Evolution of SFRD with feedback. Right panel: the solid line stands for the SFRD in halos of $T_{\text {vir }}>10^{4} \mathrm{~K}$. Other lines are the same ones as in Fig. 4. Left panel: the PopIII SFRD in halos of $T_{\text {vir }}<10^{4} \mathrm{~K}$.
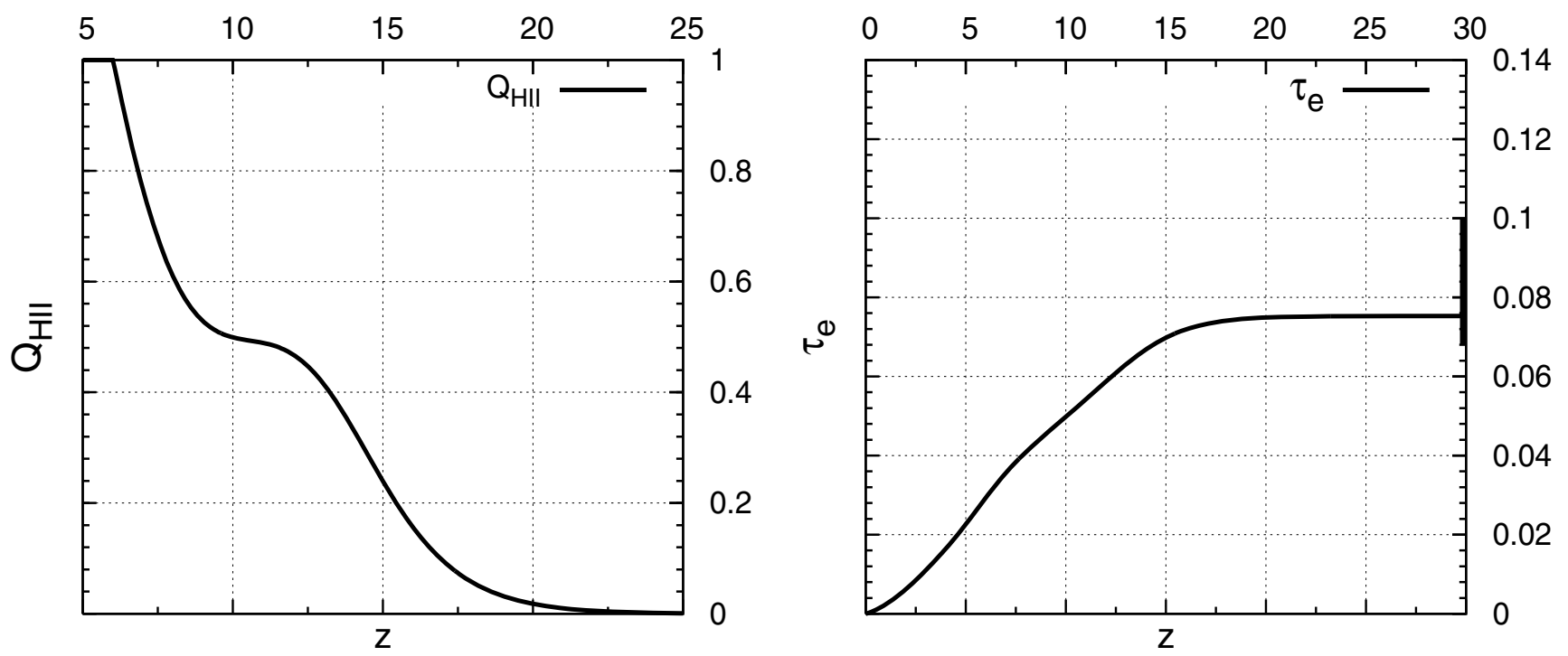

Fig. 6. Evolution of the filling factor $Q_{\mathrm{HII}}$ (left panel) and the optical depth to Thomson electron scattering $\tau_{\mathrm{e}}$ (right panel) with the redshift.

4. Although PopIII stars have high value of $f_{\mathrm{esc}}^{\mathrm{III}}$ and $n_{\gamma}^{\mathrm{III}}$, they are not able to reionize the universe considerably (about $20 \%$ at $z \sim 14)$. And the optical depth to Thomson scattering provided by PopIII stars is less than $22 \%$.

In general, we find the radiative feedback is important to the formation of the early generation stars. It suppresses the star formation considerably. But the mechanical feedback from the SNe explosions is not able to affect the early star formation significantly. The radiative and mechanical feedback dominates the star formation rate of the PopII/I stars. The feedback on SFRD from first generation stars is very strong and should not be neglected. However, their effect on the cosmic reionization is not significant, which results in a small contribution to the optical depth of electrons $\tau_{\mathrm{e}}$.
Acknowledgements. We thank Andrew Hopkins and Naveen Reddy for kindly providing the data on SFRD, Xu Kong for discussions, Li-Zhi Fang and M. Ricotti for comments. This work is partially supported by National Basic Research Program of China (2009CB824800), the National Natural Science Foundation (10733010,10673010,10573016), and the Program for New Century Excellent Talents in University.

\section{Appendix A: How to get the SFR formula with SNe feedback}

Following Cen \& Ostriker (1992), a halo with mass $M$ has the initial baryonic gas $M_{\mathrm{b}}(0)=\frac{\Omega_{\mathrm{b}}}{\Omega_{\mathrm{m}}} \times M$. Later on, partial baryonic gas condenses into cold gas $M_{\text {cold }}$. It is reasonable to assume that the rates of the mass change are proportional to $M_{\mathrm{b}}(t)$ at time $t$ :

$\frac{\mathrm{d} M_{\mathrm{b}}(t)}{\mathrm{d} t}=-M_{\mathrm{b}}(t)$ and $\frac{\mathrm{d} M_{\text {cold }}(t)}{\operatorname{det}}=+M_{\mathrm{b}}(t)$. 
This is also an implicit assumption in Cen \& Ostriker (1992). By solving Eq. (A.1) with the initial condition, we obtain

$M_{\mathrm{b}}(t)=M_{\mathrm{b}}(0) \exp (-t)$ and

$M_{\text {cold }}(t)=\int_{0}^{t} M_{\mathrm{b}}\left(t^{\prime}\right) \mathrm{d} t^{\prime}=M_{\mathrm{b}}(0)[1-\exp (-t)]$.

Cold gas can form stars with efficiency $f_{*}$. And we assume that the newly formed star mass per unit time, $\dot{M}_{\mathrm{SF}}$, is proportional to the net mass of cold gas at that time $M_{\text {cold }}^{\text {net }}(t)$ :

$\dot{M}_{\mathrm{SF}}(t)=f_{*} \times M_{\text {cold }}^{\text {net }}(t)$,

here

$M_{\text {cold }}^{\text {net }}(t)=M_{\text {cold }}(t)-f_{*}^{-1} \int_{0}^{t} \dot{M}_{\mathrm{SF}}\left(t^{\prime}\right) \mathrm{d} t^{\prime}$.

Solving Eq. (A.3) with $\dot{M}_{\mathrm{SF}}(0)=0$ (because $M_{\text {cold }}^{\text {net }}(0)=0$ ), we get

$\frac{\mathrm{d} M_{\mathrm{SF}}(t)}{\mathrm{d} t}=f_{*} M_{\mathrm{b}}(0) \times t \exp (-t)$.

If we replace $t$ with $\left[t(z)-t\left(z_{\mathrm{c}}\right)\right] /\left[\kappa t_{\mathrm{dyn}}\left(z_{\mathrm{c}}\right)\right]$, one can easily obtain Eq. (2). Since the feedback of supernovae explosions removes the cold gas at the $\operatorname{rate}^{7} \dot{M}_{\mathrm{II}}^{\mathrm{SN}}=1.1 \epsilon_{\mathrm{SN}}^{\mathrm{II}} \dot{M}_{\mathrm{SF}}(t)\left(\frac{500 \mathrm{~km} \mathrm{~s}^{-1}}{V_{\mathrm{c}}}\right)^{2}$; therefore,

$$
\begin{aligned}
M_{\text {cold }}^{\text {net }}(t) & =M_{\text {cold }}(t)-f_{*}^{-1} \int_{0}^{t} \dot{M}_{\mathrm{SF}}\left(t^{\prime}\right) \mathrm{d} t^{\prime}-\int_{0}^{t} \dot{M}_{\mathrm{II}}^{\mathrm{SN}}\left(t^{\prime}\right) \mathrm{d} t^{\prime} \\
& =M_{\text {cold }}(t)-\int_{0}^{t}\left[f_{*}^{-1}+1.1 \epsilon_{\mathrm{SN}}^{\mathrm{II}}\left(\frac{500}{V_{\mathrm{c}}}\right)^{2}\right] \dot{M}_{\mathrm{SF}}\left(t^{\prime}\right) \mathrm{d} t^{\prime},(\mathrm{A}
\end{aligned}
$$

where the circular velocity $V_{\mathrm{c}}$ is a function with two variables $M$ and $z$ (Barkana \& Loeb 2001). Then $\left\{\frac{\Omega_{\mathrm{m}}}{\Omega_{\mathrm{m}}(z)} \frac{\Delta_{\mathrm{c}}(z)}{18 \pi^{2}}\right\}^{1 / 3}$ varies between 0.65 and 1.0 when $z$ is between 30 and zero. For simplicity we use its intermediate value 0.75 in this work:

$V_{\mathrm{c}}\left(M, z_{\mathrm{c}}\right)=\sqrt{0.75} \times 23.4\left(\frac{M}{10^{8} h^{-1} M_{\odot}}\right)^{1 / 3}\left(\frac{1+z_{\mathrm{c}}}{10}\right)^{1 / 2}$.

Solving Eq. (A.3) again with $\dot{M}_{\mathrm{SF}}(0)=0$, one can get the SFR with PopII/I SNe feedback:

$$
\begin{aligned}
\frac{\mathrm{d} M_{\mathrm{SF}}^{\mathrm{II}}(t)}{\mathrm{d} t}= & \frac{M_{\mathrm{b}}(0)}{S_{\mathrm{II}}\left(M, z_{\mathrm{c}}\right)}\{\exp (-t) \\
& \left.-\exp \left\{-\left[f_{*}^{\mathrm{II}} S_{\mathrm{II}}\left(M, z_{\mathrm{c}}\right)+1\right] t\right\}\right\},
\end{aligned}
$$

where $S_{\mathrm{II}}\left(M, z_{\mathrm{c}}\right)=1.82 \times 10^{9} \epsilon_{\mathrm{SN}}^{\mathrm{II}} M^{-2 / 3}\left(1+z_{\mathrm{c}}\right)^{-1}$ for the feedback from PopII/I stars, while $S_{\mathrm{III}}\left(M, z_{\mathrm{c}}\right)=7.58 \times 10^{9} \epsilon_{\mathrm{SN}}^{\mathrm{III}} M^{-2 / 3}(1+$ $\left.z_{\mathrm{c}}\right)^{-1}$ for the feedback from PopIII stars in halos with

\footnotetext{
${ }^{7}$ In halos of mass $M \geq 10^{8} M_{\odot}$ or virial temperature of $T_{\text {vir }} \geq 10^{4} \mathrm{~K}$ after $z=30$, even a PISN with $E_{\mathrm{SN}}=10^{53} \mathrm{erg}$ cannot make a substantial gas outflow (Kitayama \& Yoshida 2005). So we assume the baryons loss in a halo should not be taken into account.
}

$T_{\text {vir }}>10^{4} \mathrm{~K}$. After substituting $\left[t(z)-t\left(z_{\mathrm{c}}\right)\right] /\left[\kappa t_{\text {dyn }}\left(z_{\mathrm{c}}\right)\right]$ for $t$, we have the SFR with SNe feedback:

$$
\begin{aligned}
\dot{M}_{\mathrm{SF}}^{\mathrm{II}}\left(M, z, z_{\mathrm{c}}\right) & =\frac{\Omega_{\mathrm{b}} M}{\Omega_{\mathrm{m}} S_{\mathrm{II}}\left(M, z_{\mathrm{c}}\right) \kappa t_{\mathrm{dyn}}}\left\{\exp \left[-\frac{t(z)-t\left(z_{\mathrm{c}}\right)}{\kappa t_{\mathrm{dyn}}\left(z_{\mathrm{c}}\right)}\right]\right. \\
& \left.-\exp \left\{-\left[f_{*}^{\mathrm{II}} S_{\mathrm{II}}\left(M, z_{\mathrm{c}}\right)+1\right] \frac{t(z)-t\left(z_{\mathrm{c}}\right)}{\kappa t_{\mathrm{dyn}}\left(z_{\mathrm{c}}\right)}\right\}\right\},
\end{aligned}
$$

and

$$
\begin{aligned}
\dot{M}_{\mathrm{SF}}^{\mathrm{III}}\left(M, z, z_{\mathrm{c}}\right) & =\frac{\Omega_{\mathrm{b}} M}{\Omega_{\mathrm{m}} S_{\mathrm{III}}\left(M, z_{\mathrm{c}}\right) \kappa t_{\mathrm{dyn}}}\left\{\exp \left[-\frac{t(z)-t\left(z_{\mathrm{c}}\right)}{\kappa t_{\mathrm{dyn}}\left(z_{\mathrm{c}}\right)}\right]\right. \\
- & \left.\exp \left\{-\left[f_{*}^{\mathrm{III}} S_{\mathrm{III}}\left(M, z_{\mathrm{c}}\right)+1\right] \frac{t(z)-t\left(z_{\mathrm{c}}\right)}{\kappa t_{\mathrm{dyn}}\left(z_{\mathrm{c}}\right)}\right\}\right\} .
\end{aligned}
$$

Obviously, Eqs. (A.9) and (A.10) reduces to Eq. (2) when $S_{\text {III }}\left(M, z_{\mathrm{c}}\right)$ approaches to zero.

\section{Appendix B: Radiative feedback from massive stars}

We focus on the radiative feedback from the ionizing background. Similar to Samui et al. (2007), we describe the strength of radiative feedback via the circular velocity of halos $V_{\mathrm{c}}$ and the fraction of ionized hydrogen $Q_{\mathrm{HII}}$. Considering the IGM in a unit volume after the ignition of the first stars, partial volume was reionized by the UV photons from massive stars in the massive halos $\left(T_{\text {vir }}>10^{4} \mathrm{~K}\right)$. For the ionized part of the halo, their SFR will be suppressed by this ionizing background, which also depends on their mass. On the other hand, the SF will continue in the rest of the volume, where the IGM is neutral, so the total SFRD is contributed by these two parts at the same time:

$$
\begin{aligned}
\dot{\rho}_{\mathrm{SF}}\left(z,>10^{4} \mathrm{~K}\right)= & \dot{\rho}_{\mathrm{SF}}^{\mathrm{HI}}(z)\left(1-Q_{\mathrm{HII}}(z)\right)+\dot{\rho}_{\mathrm{SF}}^{\mathrm{HII}}(z) Q_{\mathrm{HII}}(z) \\
= & {\left[\dot{\rho}_{\mathrm{II}}^{\mathrm{HI}}(z)+\dot{\rho}_{\mathrm{III}}^{\mathrm{HI}}\left(z,>10^{4} \mathrm{~K}\right)\right]\left(1-Q_{\mathrm{HII}}(z)\right) } \\
& +\left[\dot{\rho}_{\mathrm{II}}^{\mathrm{HII}}(z)+\dot{\rho}_{\mathrm{III}}^{\mathrm{HII}}\left(z,>10^{4} \mathrm{~K}\right)\right] Q_{\mathrm{HII}}(z) .
\end{aligned}
$$

where $\dot{\rho}_{\mathrm{II}}^{\mathrm{HI}}(z)$ stands for $\dot{\rho}_{\mathrm{II}}^{\mathrm{FB}}(z), \dot{\rho}_{\mathrm{III}}^{\mathrm{HI}}(z)$ for $\dot{\rho}_{\mathrm{III}}^{\mathrm{FB}}\left(z,>10^{4} \mathrm{~K}\right)$.

By combining Eqs. (26), (25) and (B.1), one can get,

$$
\frac{\mathrm{d} Q_{\mathrm{HII}}(z)}{\mathrm{d} z}=\Theta(z) Q_{\mathrm{HII}}(z)+\Xi(z)
$$

here,

$$
\begin{aligned}
\Theta(z)= & \frac{1}{\bar{n}_{\mathrm{H}}^{0}}\left\{n_{\gamma}^{\mathrm{III}} f_{\mathrm{esc}}^{\mathrm{III}}\left(\frac{\mathrm{d} \rho_{\mathrm{III}}^{\mathrm{HII}}\left(z,>10^{4} \mathrm{~K}\right)}{\mathrm{d} z}-\frac{\mathrm{d} \rho_{\mathrm{III}}^{\mathrm{HI}}\left(z,>10^{4} \mathrm{~K}\right)}{\mathrm{d} z}\right)\right. \\
& \left.+n_{\gamma}^{\mathrm{II}} f_{\mathrm{esc}}^{\mathrm{II}}\left(\frac{\mathrm{d} \rho_{\mathrm{II}}^{\mathrm{HII}}(z)}{\mathrm{d} z}-\frac{\mathrm{d} \rho_{\mathrm{II}}^{\mathrm{HI}}(z)}{\mathrm{d} z}\right)\right\}-\alpha_{\mathrm{B}}(1+z)^{3} \bar{n}_{\mathrm{H}}^{0} C(z) \frac{\mathrm{d} t}{\mathrm{~d} z}, \\
\Xi(z)= & \frac{1}{\bar{n}_{\mathrm{H}}^{0}}\left\{n_{\gamma}^{\mathrm{III}} f_{\mathrm{esc}}^{\mathrm{III}} \frac{\mathrm{d} \rho_{\mathrm{III}}^{\mathrm{HI}}\left(z,>10^{4} \mathrm{~K}\right)}{\mathrm{d} z}+n_{\gamma}^{\mathrm{II}} f_{\mathrm{esc}}^{\mathrm{II}} \frac{\mathrm{d} \rho_{\mathrm{II}}^{\mathrm{HI}}(z)}{\mathrm{d} z}\right\} .
\end{aligned}
$$

\section{References}

Alvarez, M. A., Bromm, V., \& Shapiro, P. R. 2006, ApJ, 639, 621 Bardeen, J. M., Bond, J. R., Kaiser, N., \& Szaly, A. S. 1986, ApJ, 304, 15 Barkana, R., \& Loeb, A. 2001, PhR, 349, 125

Barkana, R., \& Loeb, A. 2005, MNRAS, 371, 395

Bond, J. R., Arnett, W. D., \& Carr, B. J. 1984, ApJ, 280, 825 
Bouwens, R. J., Illingworth, G. D., Thompson, R. I., \& Franx, M. 2005, ApJ, 624, L5

Bouwens, R. J., Illingworth, G. D., Blakeslee, J. P., \& Franx, M. 2006, ApJ, 653, 53

Bromm, V., \& Loeb, A. 2002, ApJ, 575, 111

Bromm, V., Yoshida, N., \& Hernquist, L. 2003, Nature, 425, 812

Cen, R., \& Ostriker, P. 1992, ApJ, 339L, 113C

Chiu, W. A., \& Ostriker, J. P. 2000, ApJ, 534, 507

Choudhury, T. R., \& Srianand, R. 2002, MNRAS, 336, L27

Choudhury, T. R., \& Ferrara, A. 2006, MNRAS, 371, L55

Ciardi, B., \& Ferrara, A. 2005, SSRv, 116, 625C

Dijkstra, M., Haiman, Z., \& Loeb, A. 2004, ApJ, 613, 646

Eke, V. R., Cole, S., \& Frenk, C. S. 1996, MNRAS, 282, 263

Fan, X., Strauss, M. A., Richards, G. T., et al. 2006, AJ, 131, 1203

Fang, T., \& Cen, R. 2004, ApJ, 616, L87

Ferrarese, L. 2002, ApJ, 578, 90

Glover, S. C. O., \& Brand, P. W. J. L. 2003, MNRAS, 340, 210

Granato, G., De Zotti, G., Silva, L., Bressan, A., \& Danese, L. 2004, ApJ, 600, 580

Greif, T. H., Johnson, J. L., Bromm, V., \& Klessen, R. S. 2007, ApJ, 670, 1

Gunn, J. E., \& Peterson, B. A. 1965, ApJ, 142, 1633

Heckman, T. M., Lehnert, M. D., Strickland, D. K., \& Armus, L. 2000, ApJS, 129,493

Haiman, Z., \& Bryan, G. 2006, ApJ, 650, 7

Haiman, Z., Rees, M. J., \& Loeb, A. 1997, ApJ, 476, 458

Haiman, Z., Abel, T., \& Rees, M. J. 2000, ApJ, 534, 11

Heger, A., \& Woosley, S. E. 2002, ApJ, 567, 532

Heger, A., Fryer, C. L., Woosley, S. E., Langer, N., \& Hartmann, D. H. 2003, ApJ, 591, 288

Hopkins, A., \& Beacom, J. 2006, ApJ, 651, 142

Kitayama, T., \& Yoshida, N. 2005, ApJ, 630, 675

Komatsu, E., Dunkley, J., et al. 2008, ApJS, in press [arXiv: 0803.0547]

Lacey, C., \& Cole, S. 1993, MNRAS, 262, 627

Lapi, A., Shankar, F., Mao, J., et al. 2006, ApJ, 650, 42

Mao, J., Lapi, A., Granato, G. L., de Zotti, G., \& Danese, L. 2007, ApJ, 667, 655

Matsumoto, T., Matsuura, S., Murakami, H., et al. 2005, ApJ, 626, 31

Meynet, G., Ekstrom, S., \& Maeder, A. 2006, A\&A, 447, 623

Mo, H. J., \& White, S. D. M. 2002, MNRAS, 336, 112
Mori, M., Ferrara, A., \& Madau, P. 2002, ApJ, 571, 40

Nagamine, K., Ostriker, J. P., Fukugita, M., \& Cen, R. 2006, ApJ, 653, 881

Nadyozhin, D. K. 2003, MNRAS, 346, 97

Press, W. H., \& Schechter, P. 1974, ApJ, 187, 425

Qiu, Jing-Mei, Shu, Chi-Wang, Liu, Ji-Ren, \& Fang, Li-Zhi 2008, NewA, 13, 1

Reddy, N. A., Steidel, C. C., Pettini, M., et al. 2008, ApJS, 175, 48R

Ricotti, M., \& Ostriker, J. P. 2004a, MNRAS, 350, 539

Ricotti, M., \& Ostriker, J. P. 2004b, MNRAS, 352, 547

Ricotti, M., Gnedin, N. Y., \& Shull, J. M. 2001, ApJ, 560, 580

Ricotti, M., Gnedin, N. Y., \& Shull, J. M. 2002a, ApJ, 575, 33

Ricotti, M., Gnedin, N. Y., \& Shull, J. M. 2002b, ApJ, 575, 49

Ricotti, M., Ostriker, J. P., \& Gnedin, N. Y. 2005, MNRAS, 357, 207

Ricotti, M., Gnedin, N. Y., \& Shull, J. M. 2008, ApJ, 685, 21

Salvaterra, R., \& Ferrara, A. 2003, MNRAS, 339, 973

Salvaterra, R., Ferrara, A., \& Schneider, R. 2003, NewA, 10, 113

Salvaterra, R., Haardt, F., \& Ferrara, A. 2005, MNRAS, 362, L50

Samui, S., Srianand, R., \& Subramanian, K. 2007, MNRAS, 377, 285S

Sasaki, S. 1994, PASJ, 46, 427

Scannapieco, E., Schneider, R., \& Ferrara, A. 2003, ApJ, 589, 35S

Schaerer, D. 2003, A\&A, 397, 527

Schneider, R., Salvaterra, R., Ferrara, A., \& Ciardi, B. 2006, MNRAS, 369, 825

Seager, S., Sasselov, D. D., \& Scott, D. 1999, ApJ, 523, L1

Sheth, R. K., \& Tormen, G. 1999, MNRAS, 308, 119

Sheth, R. K., Mo, H. J., \& Tormen, G. 2001, MNRAS, 323, 1

Smith, B. D., \& Sigurdsson, S. 2007, ApJ, 661, L5

Springel, V., \& Hernquist, L. 2003, MNRAS, 339, 289

Tegmark, M., Silk, J., Rees, M. J., et al. 1997, ApJ, 474, 1

Thornton, K., Gaudlitz, M., Janka, H. T., \& Steinmetz, M. 1998, ApJ, 500, 95

Thoul, A. A., \& Weinberg, D. H. 1996, ApJ, 465, 608

White, S. D. M., \& Rees, M. 1978, MNRAS, 183, 341

Willott, C. J., Delfosse, X., Forveille, T., Delorme, P., \& Gwyn, S. D. J. 2005, ApJ, 633, 630

Wise, J. H., \& Abel, T. 2005, ApJ, 629, 615

Wyithe, J. S. B., Loeb, A., \& Carilli, C. 2005, ApJ, 628, 575

Yoshida, N., Abel, T., Hernquist, L., \& Sugiyama, N. 2003, ApJ, 592, 645

Yoshida, N., Bromm, V., \& Hernquist, L. 2004, ApJ, 605, 579

Yoshida, N., Oh, S. P., Kitayama, T., \& Hernquist, L. 2007, ApJ, 663, 687

Yu, Q., \& Lu, Y. 2005, ApJ, 620, 31 